Diabetologia 11, 27-33 (1975)

(c) by Springer-Verlag 1975

\title{
The Retinal Blood Flow in Diabetes*
}

\author{
E. M. Kohner, A. M. Hamilton, S. J. Saunders, B. A. Sutcliffe and C. J. Bulpitt \\ Department of Medicine, Royal Postgraduate Medical School, Hammersmith Hospital, London, England \\ Received: April 1, 1974, and in revised form: August 16, 1974
}

\begin{abstract}
Summary. Retinal blood flow was studied in 9 normal volunteers and 36 diabetic patients. The method used was based on the measurement of the mean transit time of flourescein in the superior temporal quandrant of the retina and on estimation of the vascular volume by measuring vessels diameters. The results showed that patients with mild or no retinopathy had significantly increased volume flow compared with normals, those with moderate retinopathy had a slight but not significant increase and those with severe retinopathy
\end{abstract}

had blood flow similar to that found in normals. The mean transit time was reduced significantly in those with mild or no retinopathy, but was similar to normals in those with moderate and severe retinopathy. Following succesful pituitary ablation and photocoagulation retinal blood flow was reduced compared with pre-treatment studies.

Key words: Diabetic retinopathy, retinal blood flow, flourescein, angiography, mean transit time.
Direct measurement of retinal blood flow is still an experimental procedure. For the study of the retinal circulation, therefore, indirect methods have to be used. One such method was described by Hickam and Frayser [1]. The method is based on the fact that in a closed vascular bed it is possible to measure the volume flow, provided the volume of the vascular bed and the circulation time of blood from arterial entry to venous exit is known:

$$
\text { Volume flow }=\frac{\text { volume of vascular bed }}{\text { mean circulation time, }}
$$

The present paper reports a study using a modification [2] of Hickam and Frayser's [1] method to estimate the volume flow of the superior temporal quadrant in diabetic patients.

\section{Materials and Methods}

\section{Patients}

Nine normal volunteers and 36 diabetic patients were studied on one or more occasions.

The normal volunteers were either members of the hospital staff (5) or patients who attended for flourescein angiography and who had no lesions in the eye studied (4).

Diabetic patients were selected from the Diabetic Clinic at the Hammersmith Hospital. Of a total of 96 patients who had flourescein angiograms for this purpose, only 36 were suitable for extimation of the

* This work was supported by the Wellcome Trust and The British Diabetic Association. retinal blood flow, because the quality of the angiograms and the distribution of the retinal vessels were suitable for the study (see below).

The diabetic patients were subdivided into 3 groups: those with no or only 'mild' retinopathy (14 patients, 2 of these were newly discovered diabetics, the others had their disease for over 5 years), those with 'moderate' retinopathy (10 patients), including the earliest forms of neovascularisation, and those with severe proliferative lesions ( 12 patients). The subdivision was made on the basis of retinal photographs by an ophthalmologist (A. M. H.).

In the normals one eye only was studied while in 6 diabetic patients the transit time was measured in both eyes.

\section{Methods}

a) Measurement of Transit Time. Flourescein angiography can be used for the measurement of the mean transit time as Hickam and Frayser [1] have shown, when $4-5 \mathrm{ml}$ of $5 \%$ flourescein are injected into a peripheral vein, the flourescence emitted from the retinal vessels is proportional to the concentration of dye in those vessels. It has also been shown that if the correct film is used and care is taken with its development, so that it has a $y$ of 1 [3] then the antilog of the image density (minus the antilog of the baseline density) is proportional to the intensity of the flourescence $\left(I_{f}\right)$.

The method used was that described by Bulpitt and Dollery [2] with slight modifications. Retinal photographs were taken using the Zeiss (Oberkochen) 
camera and an automatic Nikon camera back. The Nikon camera was modified to house a timing device which photographed the time to the nearest $0.1 \mathrm{sec}$ onto the film. For the flourescein angiograms the Baird Atomic (B 5) interference filter and Kodak No. 300976 glass barrier filter were used. Kodak Tri X film was exposed to flash 2 (120 joules) and developed in D 76 developer at room temperature (approximately $18-20^{\circ} \mathrm{C}$ ) for $8^{1 / 2} \mathrm{~min}$, thus giving a $\gamma$ of 1 .

Flourescein angiograms were taken after injection of $4 \mathrm{ml}$ of $5 \%$ sodium flourescein through an indwelling cathether. Baseline pictures were taken before injection of the dye and at approximately one second intervals after its appearance, until recirculation became visible.

The optical density of the superior temporal vessels was measured from the photographic negatives using a Joyce-Loebel double beam recording densitometer. The densitometer was calibrated using neutral density filters. The vessels selected were scanned as near to the disc as possible both on the baseline negative and later on each negative throughout the passage of dye through the superior temporal quadrant.

The area obtained under the density curve was measured by an integrator fitted to the densitometer. The base of each curve was measured. The mean optical density of the artery and vein could therefore be calculated for each frame. The intensity of flourescence was computed from this and plotted against time on semi-logarithmic paper. The downslope of this line was usually linear until the recirculation of the flourescein (Fig. 1). From the points prior to recirculation the straight line to $I_{f} \times 10=1$ was computed on the 'least squares' method [2].

The mean transit time for both the artery and the vein was calculated from the formula.

$$
t=\frac{\sum_{n}^{o} I_{f}(t) t}{\sum_{n}^{o} I_{f}(t)}
$$

where $I_{f}(t)=$ the intensity of flourescence at time $t$, from time 0 to time $n, n$ being the time when $I_{f}=1$. The mean circulation time is the difference between the venous and arterial transit times.

b) Estimation of Vascular Volume. It is not possible to measure the vascular volume, but it can be estimated. The following assumptions were therefore made:

1. The superior temporal vessels supply the entire area of the segment considered, i.e., all the blood reaching the area has to flow through the superior temporal artery, and the entire drainage is through the superior temporal vein. The area therefore represents a closed vascular bed. This is never entirely true, since there is always some anastomosis with neighbouring vessels. However, to minimise any error only those eyes were included in the study in which the superior temporal vessels supplied an area the linear borders of which subtended an angle of at least $75^{\circ}$ at the disc. The arterial and venous overlap had to be at least $80 \%$ of this area.

2. Since in most organs about $76 \%$ of the blood volume is in the veins and $21 \%$ in the arteries and only $3 \%$ in the capillaries [4] the volume of the capillary bed can be ignored.

\section{G.W. L. Superior temporal vessels}

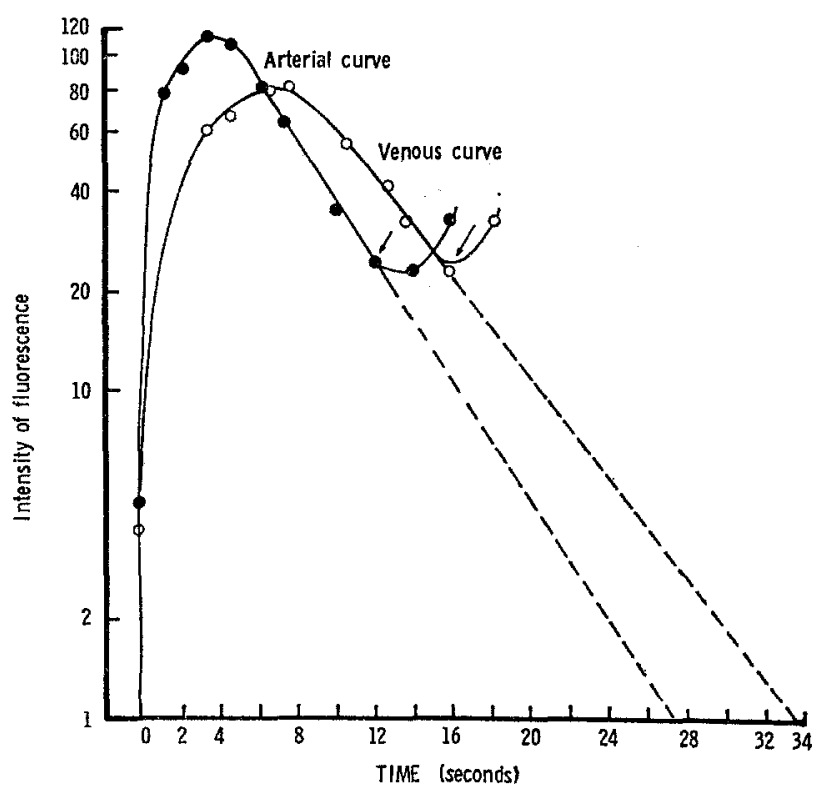

Fig. 1. Intensity of flourescence on logarithmic scale plotted against time for the superior temporal vessels in patient $G$. W. Note linear downslope until recirculation of dye, indicated by arrows

3. In spite of the variable branching pattern of the retinal vessels from eye to eye, the length of the vessels is similar in different eyes. To ascertain this as far as possible, refractions of more than \pm 2 Dioptres were excluded.

4. The cross sectional area of the feeding artery reflects the blood volume contained in the arteries and similarly the cross sectional area of the draining vein reflects the blood contained in the veins. Finally, it is also assumed that the cross section of these vessels is a circle.

That these assumptions were reasonable was shown 
by Hickam and Frayser [1] and by Bulpitt and Dollery [2]. The vascular volume was therefore estimated in this study from measuring the vessel diameters at the site of scanning with the densitometer. To obtain the true internal diameter of the vessels [5] the diameters were measured from those frames on the flourescein angiograms showing maximal filling of the vessels, using a Vanguard motion picture analyser.

c) Estimation of Volume Flow. The volume flow was calculated by measuring the mean transit time for artery and vein as described, and estimating the vascular volume from the cross sectional area of the vessels. The results are expressed in arbitrary units.

d) Analysis. Since the majority of patients had only one eye photographed, in those who had both eyes studied the mean of the two eyes was taken for analysis. Several patients and three normal volunteers had more than one study. For the main analysis the mean of these was taken, unless the patient had treatment between the initial and subsequent examinations. Separate analysis was performed to show changes in flow and transit time between the initial and subsequent studies.

\section{Results}

a) Volume Flow. Fig. 2 shows the volume flow in normals and the 3 groups of diabetic patients.

In the normal group the mean figure was $8.5 \times 10^{3}$ arbitrary units (AU) and the range $4.8-11.9 \times 10^{3}$ AU. In the diabetic patients the volume flow was greater than in the normals, mean: $13.7 \times 10^{3} \mathrm{AU}$ and the range much larger $\left(2.9-37.5 \times 10^{3} \mathrm{AU}\right)$. There was considerable overlap between normals and diabetics. The difference for the group as a whole was not significant. However, when the diabetic patients were subdivided according to the severity of the retinopathy the situation was different. Those with the most severe lesions had blood flow similar to normals with relatively small increase in range (mean: 9.5, range $4.6-19.2 \times 10^{3} \mathrm{AU}$ ). In those with moderate retinopathy the mean flow was nearly double that seen in normals, but the difference was not significant because of the large standard deviation. Those with no or only mild retinopathy had a volume flow significantly higher than normals $(p=0.02)$ with a mean of 16.3 and a range of $8.2-37.5 \times 10^{3} \mathrm{AU}$.

Table 1

\begin{tabular}{|c|c|c|c|c|}
\hline & \multirow[b]{2}{*}{ Normal } & \multicolumn{2}{|l|}{ Retinopathy } & \multirow[b]{2}{*}{ Severe } \\
\hline & & Mild + None & Moderate & \\
\hline No. of Patients & 9 & 14 & 10 & 12 \\
\hline No. of Eyes & 9 & 14 & 11 & 15 \\
\hline No. Re-studied & 3 & 3 & $4(2)^{a}$ & $12(10)^{\mathrm{a}}$ \\
\hline $\begin{array}{l}\text { Age (years) } \\
\text { Mean and range }\end{array}$ & $\begin{array}{l}39.4 \\
(28-62)\end{array}$ & $\begin{array}{l}45.2 \\
(26-66)\end{array}$ & $\begin{array}{l}51.7 \\
(36-67)\end{array}$ & $\begin{array}{l}40.8 \\
(29-56)\end{array}$ \\
\hline $\begin{array}{l}\text { Mean Blood Pressure } \\
(\mathrm{mm} \mathrm{Hg}) \\
\text { Mean } \pm \text { s.d. }\end{array}$ & $95.7 \pm 12.9$ & $90.8 \pm 13.1$ & $113.9 \pm 16.8$ & $98.7 \pm 16.5$ \\
\hline $\begin{array}{l}\text { Packed cell volume } \\
(\%) \\
\text { Mean } \pm \text { s.d. }\end{array}$ & $41.8 \pm 5.1$ & $40.4 \pm 5.2$ & $39.3 \pm 4.2$ & $42.7 \pm 2.8$ \\
\hline $\begin{array}{l}\text { Blood sugar } \\
(\mathrm{mg} \%) \\
\text { Mean } \pm \text { s.d. }\end{array}$ & - & $149.5 \pm 79.5$ & $169.0 \pm 87.8$ & $195.3 \pm 90.5$ \\
\hline
\end{tabular}

a Numbers in brackets indicate those studied pre- and post treatment

The Statistical method used for comparing the groups was the student's ' $t$ ' test for unpaired samples. The ' $t$ ' test for paired samples (with correction for small numbers) was used when the volume flow in the same patient was studied at various times or before and after treatment. All studies were considered when correlating blood flow with mean blood pressure, packed cell volume and blood sugar, provided the patient had no specific treatment for the retinopathy. Summary of the patient details are shown in Table 1. b) Vascular Volume and Mean Transit Time. Volume flow increase must be due to either increase in vascular volume or a decrease in transit time. In this group of patients both the arterial and the venous diameters were slightly larger in the diabetics than in the normals, but the difference was not significant because of the large overlap.

The mean transit time was significantly less in those diabetics with mild or no retinopathy $(p<0.01)$ than in the normals and their range was similar (Fig. 3). 
Patients with moderate and severe retinopathy had mean transit times similar to that seen in normals.

c) Repeatability. Three normals and six diabetic patients had more than one study (Fig. 4). While the diabetic patients tended to have a greater variability than the normals both in the transit time and the volume flow the differences were not significant.

d) Effect of Treatment. Fig. 5 and 6 show the effect of treatment by pituitary ablation and photocoagulation on volume flow and on mean transit time. Of the 6 patients who had Yttrium-90 implantation of the pituitary, three showed marked reduction of the blood

SUPERIOR TEMPORAL VOLUME FLOW

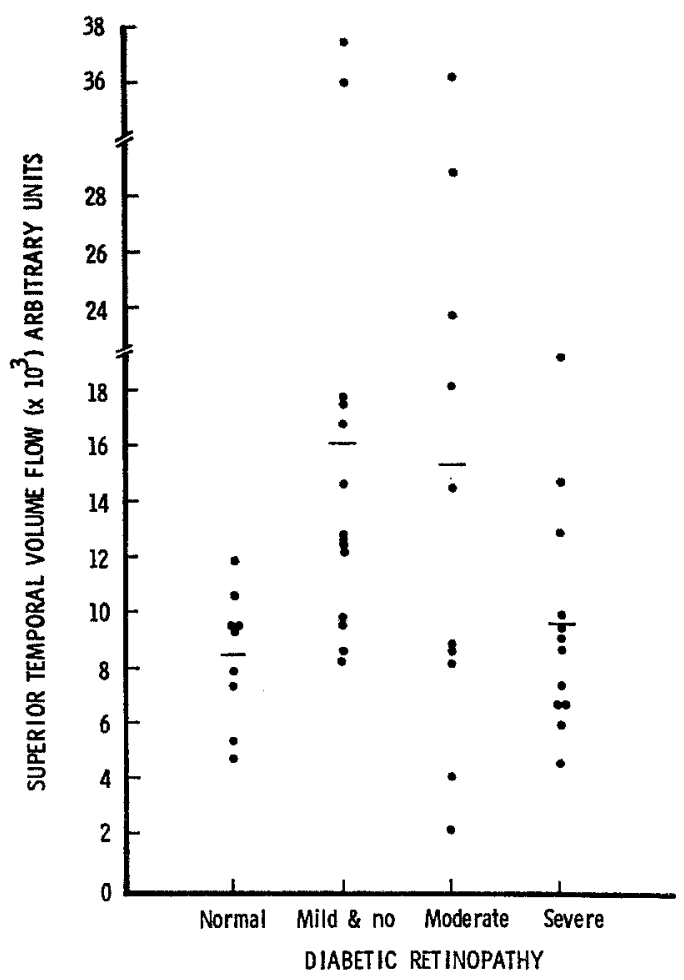

Fig. 2. Superior temporal volume flow in 9 normal volunteers and 36 diabetic patients subdivided into those with mild and no retinopathy, moderate retinopathy and severe retinopathy. Horizontal lines indicate mean values

flow; these were the patients in whom the retinopathy showed the greatest regression. In all 6 patients who had photocoagulation the volume flow was reduced compared with pre-treatment studies. But even in this group there was only a trend and no statistically significant difference between the pre and post treatment studies $(p=0.07)$.

The transit times tended to change in the opposite direction to blood flow, but the greatest change in one did not necessarily indicate the greatest change in the other, i.e. there was no inverse correlation between the two.

e) Effect of Other Parameters. There was no correlation between volume flow and blood pressure, packed cell volume, or blood sugar, whether the group was taken as a whole or when subdivided according to severity of the retinopathy.

\section{Discussion}

There are no previous papers on the measurements of retinal blood flow in diabetic patients (preliminary

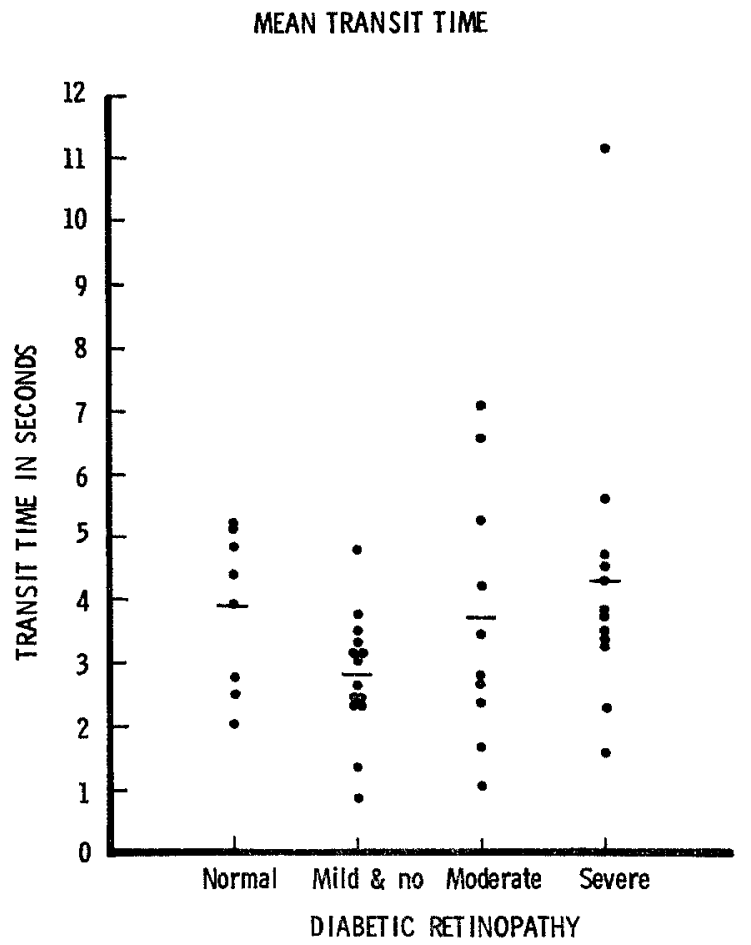

Fig. 3. Mean transit time of flourescein in superior temporal vessels in the same patients as shown in Fig. 2. Horizontal levels indicate mean values

reports of the present work were published by Kohner [6], and Kohner et al. [7]). On the basis of clinical observations Davis [8] and Goldberg [9] suggested that retinal blood flow is reduced in diabetic retinopathy. The findings reported in this paper showing a great variability in blood flow between individual patients and even in the same patient studied at different times and between groups of patients with differing severity of retinopathy was totally unexpected when the study was started.

In an attempt to elucidate the causes of variability in blood flow three parameters - packed cell volume, blood pressure and blood sugar - were studied. 
VOLUME FLOW TRANSIT TIME
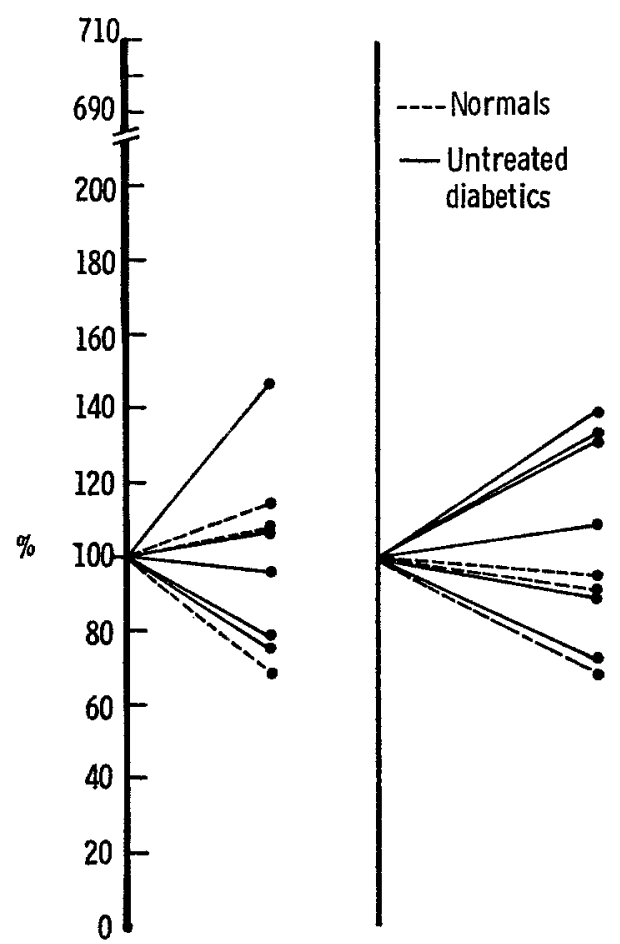

Fig. 4. Percentage change in volume flow and transit time in 3 normals (interrupted line) and 6 diabetic patients (solid lines) restudied between 6 months and 1 year after initial study. Initial study designated as $100 \%$

\section{PITUITARY ABLATED PHOTOCOAGULATED}

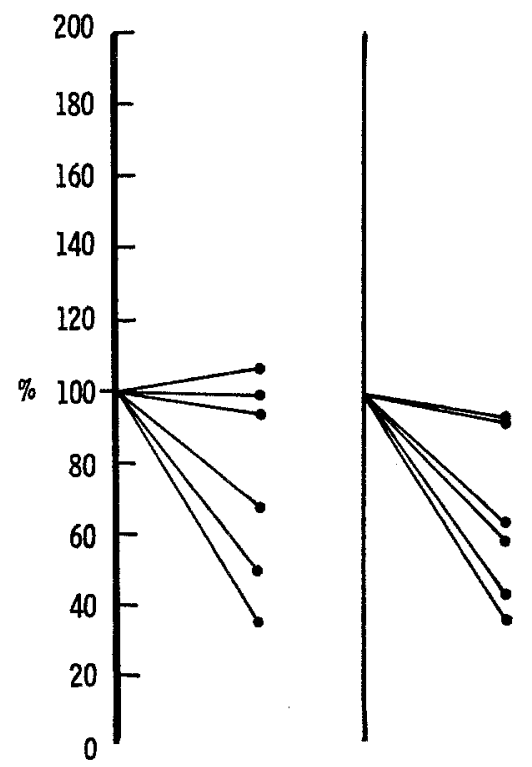

Fig. 5. Percentage reduction in volume flow in patients treated by pituitary ablation and photocoagulation. Pre-treatment value indicated as $100 \%$

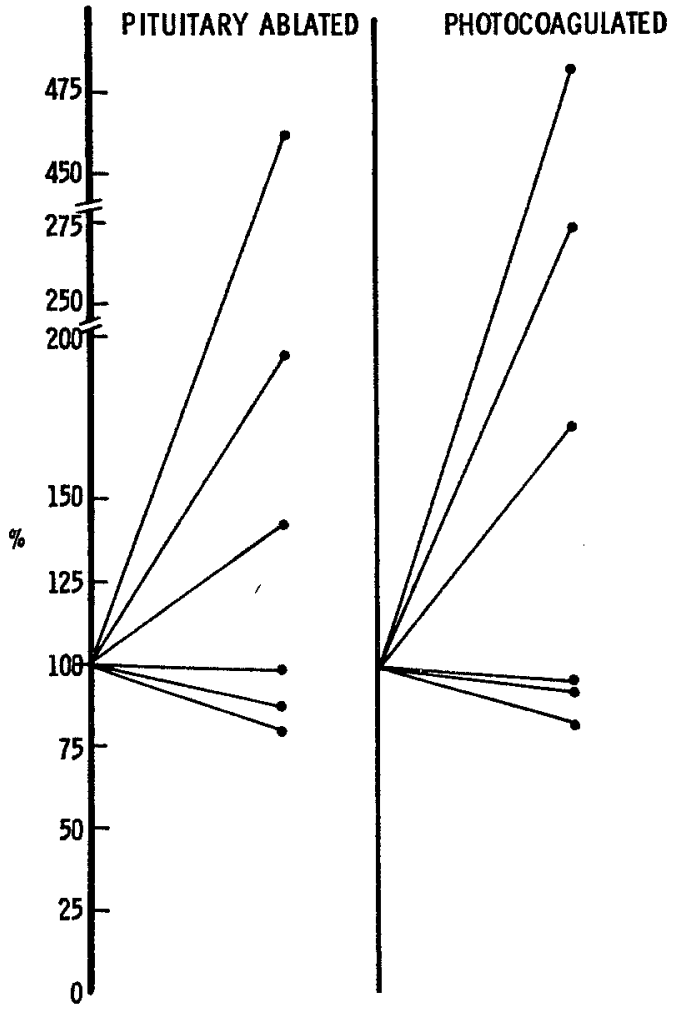

Fig. 6. Percentage increase in transit time in patients treated by pituitary ablation and photocoagulation. Pre-treatment value indicated as $100 \%$

It has been shown by Bulpitt and Dollery [2] that retinal blood flow is increased in anaemia. While the packed cell volume in the diabetic patients was lower than in normals the difference was not significant, nor was there a correlation between packed cell volume and retinal blood flow in the diabetic patients. There was also no correlation between blood pressure and retinal volume flow, therefore this parameter could not account for the variability observed in the diabetic population. This finding is similar to that observed in normals by Bulpitt and Dollery [2] but is in contrast to the findings of Archer (unpublished observations) in hypertensive patients where higher blood pressure was associated with increased volume flow in the patients restudied at different times (even in the absence of hypertensive retinopathy).

In diabetes blood sugar levels would be expected to have an influence on the volume flow, reflecting the metabolic state. Since no correlation was found between blood sugar levels and volume flow the great variation in flow seen in these patients cannot be attributed to this. It must however be emphasised that most of the studies were carried out in the early afternoon $1 / 2-2$ hrs post prandially when most of the pa- 
tients had rather high sugar levels. It is possible that an estimate of the metabolic state based on the blood sugar level during the $24-48 \mathrm{hrs}$ period before the volume flow determination would have shown a correlation between retinal blood flow and degree of metabolic decompensation.

No single factor measured seems to account for the variability in blood flow seen in diabetic patients, but it is noteworthy that the variation was much less in those with severe retinopathy indicating the possibility that local factors may be of greatest importance.

Why, then, is retinal blood flow increased in diabetes and are the difficulties in measurement encountered likely to invalidate the results?

One of the difficulties in this study was the leakage of flourescein from microvascular abnormalities. This could alter the mean transit time, since the volume in which flourescein is distributed will increase, and thus those with the most advanced retinopathy will have a slower transit time (as seen in this study). This explanation is unlikely because leakage of flourescein out of vessels is rapid, its reabsorption on the other hand takes over 24 hrs. The measurements made here during the first transit of dye only would result in a shortened, rather than longer transit time. To minimise this possible source of error patients with significant new vessels in the superior temporal area and those with the worst leakage from arteries and veins were excluded.

The formation of new vessels could account in some instances for increased blood flow, if the flow through these vessels was excessive. In this case those with the severest retinopathy would have had the most marked increase of volume flow, rather than those with mild or no retinopathy.

The volume flow and mean transit time in those with severe retinopathy was similar to that seen in normals. It must, however, be noted that most of these patients had large areas of peripheral non perfusion, involving not only capillary bed but small arterioles and veins. The vascular volume was therefore reduced. The 'normal' blood flow may indicate a relatively large flow through a smaller vascular volume. The arterial and venous diameters would not necessarily reflect this if the vessel wall was diseased and allowed the entry of flourescein thus giving an artificially wide diameter.

It could indeed be argued that when the vascular bed is as abnormal as in severe retinopathy the method used is not really valid.

Increased volume flow in other organs of diabetics has been reported, by Butterfield and Wichelow [10] Alexander and his collegues [11] and Christensen [12] and others. Findings similar to those in this work were reported in abstract form by Christacopoulos et al. [13] who measured the transit time of flourescein in the retina in pre-diabetic and chemical-diabetic patients and found it to be shorter than in normals. They explain their findings by the presence of arteriovenous shunts in the retinal periphery in prediabetes. Such shunts have never been demonstrated clinically.

The area under the venous curves was in the normals $20-30 \%$ greater than the arterial area. Hickam Frayser [1] explain this by the streaming of flourescein in the veins. In diabetics the variation was greater but the findings were similar. The downslope of both the arterial and venous curves tended to be steep, falling towards zero more rapidly than in normals indicating some reduction in peripheral resistance. These studies thus do not exclude the possibility of arterio-venous shunts. If such shunts existed they would cause diversion of blood from areas of the capillary bed, causing non perfusion of large areas in early diabetes and mild retinopathy. A more likely explanation is generalised dilation of the capillary bed (as noted by Vink [14]) indicating generally reduced peripheral resistance. This could be the result of autoregulation in the retinal circulation. (By autoregulation in this context we meant that blood flow through an organ is regulated according to the metabolic needs of that organ, as defined by Guyton and his colleagues [15]). Both lack of an essential energy source or accumulation of a metabolic end product could act as an autoregulatory stimulus. While experimental work indicates that insulin is probably not essential for retinal metabolism of carbohydrates, increasing blood glucose concentration in the alloxan diabetic rats resulted in conspicuous rise in retinal lactate levels [16]. A fall in $\mathrm{pH}$ which accompanies such metabolic change would be expected to cause an increase in blood flow.

Little is known about the metabolism of the retina but the most potent stimulant of autoregulation has been shown to be variation in the arterial $\mathrm{pO}_{2}[17,18]$. Clearly arterial $\mathrm{pO}_{2}$ is unlikely to be abnormal in early diabetes and in those with mild retinopathy. However Ditzel [19] and Standl and Kolb [20] showed that in early and poorly controlled diabetic patients the level of the erythrocyte 2,3 - diphosphoglycerate level is reduced. This would result in a shift of the oxygen dissociation curve to the left. Dietzel [19] estimates that the levels found in his patients would result in a 30\% decrease of available oxygen at a $\mathrm{pO}_{2}$ of $40 \mathrm{~mm}$ (the venous end of the capillary bed). Thus relative hypoxia could exist, accounting for vascular dilatation. This is most marked in the resistance vessels, the diameters of which were not measured in the present experiments and would only be reflexted in an: increased blood flow. 
If diabetic retinae required increased amounts of oxygen as indicated by some workers [21, 22] the stimulus would be even greater. This hypothesis though probably too simple is also attractive because it explains the poor response of retinal vessels of diabetic patients to changes in blood gases [23]. The lack of increased blood flow in those with severe retinopathy could be explained on the basis that the blood vessels are so diseased by this time that they cannot respond even to a strong autoregulatory stimulus.

Clearly more work on both retinal blood flow and retinal metabolism will have to be done before all the changes found in diatbetes can be explained.

Acknowledgements. We gratefully acknowledge the helpful discussions and advice of Professor C. T. Dollery. We are also grateful to Professor T. Russel Fraser for allowing us to study patients under his care.

\section{References}

1. Hickam, J. B., Frayser, R.: A photographic method for measuring the mean retinal circulation time using flourescein. Invest. Ophthal. 4, 876-884 (1965)

2. Bulpitt, C. J., Dollery, C. T.: The estimation of retinal blood flow by measurement of the mean circulation time. Cardiovase. Res. 3, 406-412 (1971)

3. Wilsey, R. B.: Photography: sensitometry and densitometry. In: Medical physics. Vol. I. Chicago: Yearbook Publishing Co. 1944

4. Litton, A.: Anatomical factors affecting microcirculation. In: The William Mackenzie Centenary Symposium on the Ocular Circulation in Health and Disease (ed. Cant, St.) pp. 18-25. Kimpton: 1969

5. Bulpitt, C. J., Dollery, C. T., Kohner, E. M.: The marginal plasma zone in the retinal microcirculation. Cardiovasc. Res. 4, 207-212 (1970)

6. Kohner, E. M.: The effect of diabetes on retino-vascular function. Acta diabet. lat. 8, (Supp1. 1) 135-176 (1971)

7. Kohner, E. M., Sutcliffe, B. A., Tudball, M., Dollery, C. T. The retinal blood flow in diabetes. Diabetologia 9,75 (1973)

8. Davis, D. D.: Natural course of diabetic retinopathy. In: Vascular complications of diabetes mellitus (eds. Kimura and Caygill) pp. 139-169. Mosby Co. 1967

9. Goldberg, M.: In: Diabetic retinopathy (eds. Lynn, J. R., Snyder, W. B., and Vaiser, A.) New York: Grune and Stratton Inc. (in press)
10. Butterfield, W. H., Wichelow, M.S.: Peripheral glucose metabolism in control subjects and diabetic patients during glucose, glucose-insulin and insulin sensitivity tests. Diabetologia 1, 45-53 (1965)

11. Alexander, K., Teusen, R., Mitzkat, H. J.: Vergleichende Messungen der Extremitätdurchblutung bei Diabetikern und Stoffwechselgesunden. Klin. Wschr. 46, 234-238 (1968)

12. Christensen, N. J.: A reversible vascular abnormality associated with diabetic ketosis. Clin. Sci. 39, 539 (1970)

13. Christocopoulos, P. D., Soeldner, J. S., Gleason, R. E.: Variation in retinal haemodynamics in different stages of diabetes. Excerpta med. (Amst.) International Congress Series 280, 180-181 (1973)

14. Vink, R.: Flourescein angiography in diabetic retinopathy. Thesis, pp. 22-23. Leiden 1969

15. Guyton, A. C., Ross, J. M., Carrier, O., Walker, J. R.: Evidence for tissue oxygen demand as the major factor causing autoregulation. Circulat. Res. Suppl. 1, 14-15 (1964)

16. Keen, H., Chlouverakis, C.: Metabolic factors in diabetic retinopathy. In: Biochemistry of the Retina (ed. Graymore, C. N.pp. 123-136. Academic Press 1965

17. Sicker, J. O., Hickam, J. B.: Normal and impaired vascular reactivity. Circulation 7, 79-82 (1953)

18. Bulpitt, C. J., Kohner, E. M., Dollery, C. T.: Autoregulation in the retinal microcirculation. VI European Conference on Microcirculation 1970. pp. 96-100 (eds. Ditzel, J. and Lewis, D. H., Basel: S. Karger 1971

19. Ditzel, J.: Impaired oxygen release caused by alterations of the metabolism in the erythrocytes in diabetes. Lancet 1972 I, $721-722$

20. Standl, E., Kolb, H. J.: 23 diphosphoglycerate fluctuations in erythrocytes reflecting pronounced blood glucose variation. Diabetologia 9, 461-466 (1973)

21. Illing, E. K. B., Gray, C. H.: Retinal metabolism in diabetes: Metabolism of retinae of normal and alloxan-diabetic rabbits. J. Endocr. 7, 242-247 (1951)

22. DeRoetth, A. J., Pei, Y. F.: Metabolism of the alloxan diabetic rat retina. Arch. Aphthal. 71, 73-76 (1964)

23. Hickam, B. Sieker, J. O.: Retinal vascular reactivity in patients with diabetes mellitus and with atherosclerosis. Circulation 22, 243-246 (1960)

E. M. Kohner, M. D.

Royal Postgraduate Medical School

Hammersmith Hospital

London W 12

England 